\title{
ON THE FORMULAE FOR CALCULATING THE MAGNETIC FIELD IN THE SOURCE OF SOLAR MICROWAVE BURSTS
}

\author{
REN-YANG ZHAO \\ Beijing Observatory, Academia Sinica, Beijing 100080, China
}

\begin{abstract}
Based on the theory of gyro-synchrotron radiation from both thermal and nonthermal electrons, and adopting the relation between the peak frequency and the corresponding rise time of microwave bursts, we have derived several analytical formulae for calculating the magnetic field in the source regions of solar microwave bursts.
\end{abstract}

\section{INTRODUCTION}

Solar microwave bursts associated with magnetic fields are principally impulsive bursts. The radiation mechanism for these bursts is gyro-synchrotron emission from thermal electrons having a Maxwellian distribution or nonthermal electrons with a power-law distribution. The magnetic field, which is an important physical parameter of active region plasma, plays a decisive role in the emission mechanism of gyro-synchrotron radiation of microwave bursts. For example, the magnetic field strength directly determines the radiation frequency, and is closely related to the emissivity and the absorption coefficient which are the two most essential physical parameters of radiation theory.

The magnetic field is both an essential and important key physical parameter for researching microwave bursts. The magnetic field in the corona above the photosphere, however, cannot be directly measured, so it is necessary to seek ways to derive the magnetic field in the source region of microwave bursts above the photosphere. In the present paper we use the relation between the peak frequency and the corresponding rise time of microwave bursts (Zhao, Magun, \& Schanda 1990), according to the mechanism of gyro-synchrotron radiation from both thermal and nonthermal electrons, and have derived several analytical formulae for calculating the magnetic field in the source region of microwave bursts under the conditions of thermal and nonthermal models.

\section{FORMULAE OF MAGNETIC FIELD}

Peak Frequency and Rise Time

The rise time of a microwave burst has a certain theoretical significance because it reflects the energy release process in flare regions. Recently, many scientists have studied the rise time and other relevant physical parameters of the microwave bursts, by using thermal and nonthermal models, e.g., Brown, Melrose, \& Spicer (1979), Smith \& Lilliequist (1979), Batchelor et al. (1985), and Zhao, 
Magun, \& Schanda (1990).

Using observational data of microwave bursts, Zhao, Magun, \& Schanda (1990) have obtained a power-law relation between the peak frequency, $f_{p}$, and the corresponding rise time, $t_{r}$, of microwave bursts as follows:

$$
f_{p} \approx 1.517 \times 10^{10} t_{r}^{-0.14}
$$

where $f_{p}$ is in units of $\mathrm{Hz}$ and $t_{r}$ in units of sec.

Formulae of Magnetic Field for a Thermal Model

On the basis of the model considered by Zhao, Magun, \& Schanda (1990), the theoretical rise time of the microwave bursts can be defined as follows:

$$
t_{r} \approx L / c_{s},
$$

where $L$ is the half length of the loop and $c_{s}$ is the ion-acoustic speed. Let $\sigma$ be the ratio of the total length, $2 L$, to the mean width, $W$, of the loop, then

$$
\sigma \approx 2 L / W,
$$

and let the central height $h$ of the loop be approximately equal to its half length $L$, so that

$$
h \approx L \text {. }
$$

The peak frequency of the gyro-synchrotron radiation in the range of harmonics $\approx 10$ to $\approx 100$ from thermal electrons with a temperature range of $\approx 5 \times 10^{7}$ to $\approx 5 \times 10^{9} \mathrm{~K}$ can be approximated as (Dulk 1985):

$$
f_{p} \approx \begin{cases}1.4\left(N_{e} W / B\right)^{0.1}(\sin \theta)^{0.6} T_{e}^{0.7} B & \left(10^{8}<T_{e}<10^{9} K\right) \\ 475\left(N_{e} W / B\right)^{0.05}(\sin \theta)^{0.6} T_{e}^{0.5} B & \left(10^{7} \leq T_{e} \leq 10^{8} K\right),\end{cases}
$$

where $\theta$ is the viewing angle, $B$ the magnetic field strength, $T_{e}$ the electron temperature, and $N_{e}$ the electron density in the source regions of the microwave bursts. Generally, the valid range of $\theta$ can be taken as $10^{\circ} \leq \theta \leq 80^{\circ}$. Therefore, combining equations (1) to (5), we have derived the analytical expressions of the magnetic field formulae as follows:

$$
B \approx \begin{cases}5.40 \times 10^{11} \sigma^{0.11}(\sin \theta)^{-0.67} N_{e}^{-0.11} T_{e}^{-0.70} h^{-0.27} & \left(10^{8}<T_{e}<10^{9} K\right) \\ 2.93 \times 10^{8} \sigma^{0.05}(\sin \theta)^{-0.63} N_{e}^{-0.05} T_{e}^{-0.45} h^{-0.20} & \left(10^{7} \leq T_{e} \leq 10^{8} K\right)\end{cases}
$$

It can be seen from equation (6) that $B$ depends on $T_{e}$ sensitively, but is not sensitive to $N_{e}$ and $\sigma$. Therefore, by taking $\sigma=5$, for the typical values of $N_{e}$ (e.g. $10^{9}, 10^{10}$ and $10^{11} \mathrm{~cm}^{-3}$ ) and $\theta\left(\right.$ e.g. $45^{\circ}$ and $75^{\circ}$ ), using equation (6), then one can calculate the magnetic field $B$ in the source regions of microwave bursts at height $h$ for given values of $T_{e}$ (e.g. $2 \times 10^{7}, 5 \times 10^{7}, 8 \times 10^{7}, 2 \times 10^{8}, 5 \times 10^{8}$, and $8 \times 10^{8} \mathrm{~K}$ ).

As for the electron temperature and density, they can be derived from the X-ray data (Dulk, Melrose, \& White 1979). For example, the photon spectrum of a hard X-ray, $I(E)$, can be written as (e.g. Crannell et al. 1978)

$$
I(E)=1.3 \times 10^{3}(E M)_{45} E^{-1.4} T^{-0.1} \exp (-E / T),
$$


where $I(E)$ is in units of photons $\mathrm{cm}^{-2} \mathrm{~s}^{-1} \mathrm{keV}^{-1}$, the photon energy $E$ and the temperature $T$ are in units of $\mathrm{keV}$, and the emission measure $(E M)_{45}$ (in units of $10^{45} \mathrm{~cm}^{-3}$ ) is given by

$$
(E M)_{45}=0.1 N_{10}^{2} W_{8}^{3},
$$

where the density, $N_{10}$, is in units of $10^{10} \mathrm{~cm}^{-3}$, and the scale length, $W_{8}$ in units of $10^{8} \mathrm{~cm}$. Another useful relation comes from the microwave flux density, $S$, which, for $f \leq f_{p}$, is given by the Rayleigh-Jeans law for an optically thick source. It can be written as (Dulk, Melrose, \& White 1979)

$$
S \approx 1.36 \times 10^{-2} T_{8} f_{9}^{2} W_{8}^{2},
$$

where $S$ is in units of s.f.u., and $f_{9}$ is the frequency in GHz. Using equations (7) - (9), one can calculate the electron temperature, $T_{e}$, and the electron density, $N_{e}$, from the X-ray spectrum and the radio spectrum of the microwave bursts.

According to equation (5), we can directly write another expression for the magnetic field :

$$
B \approx \begin{cases}0.69 W^{-0.11}(\sin \theta)^{-0.67} N_{e}^{-0.11} T_{e}^{-0.78} f_{p}^{1.11} & \left(10^{8}<T_{e}<10^{9} K\right) \\ 1.52 \times 10^{-3} W^{-0.05}(\sin \theta)^{-0.63} N_{e}^{-0.05} T_{e}^{-0.53} f_{p}^{1.05} & \left(10^{7} \leq T_{e} \leq 10^{8} K\right) .\end{cases}
$$

If the relation between $f_{p}$ and $t_{r}$ is also considered from equations (1) and (5) we can give a third expression for the magnetic field:

$$
B \approx \begin{cases}1.41 \times 10^{11} W^{-0.11}(\sin \theta)^{-0.67} N_{e}^{-0.11} T_{e}^{-0.78} t_{r}^{-0.16} & \left(10^{8}<T_{e}<10^{9} K\right) \\ 7.93 \times 10^{7} W^{-0.05}(\sin \theta)^{-0.63} N_{e}^{-0.05} T_{e}^{-0.53} t_{r}^{-0.15} & \left(10^{7} \leq T_{e} \leq 10^{8} K\right) .\end{cases}
$$

In consequence, if the peak frequency or the corresponding rise time is obtained by means of observations, then the magnetic field in the source regions of microwave bursts may be calculated using equations (10) or (11).

Formulae of Magnetic Field for a Nonthermal Model

The peak frequency of the gyro-synchrotron radiation in the range of harmonics $\approx 10$ to $\approx 100$ from nonthermal electrons can be approximated as (Dulk 1985):

$$
f_{p} \approx 2.72 \times 10^{3} 10^{0.27 \delta}(\sin \theta)^{0.41+0.03 \delta}\left(N_{e} W\right)^{0.32-0.03 \delta} B^{0.68+0.03 \delta},
$$

where $\delta$ is the electron energy index. The valid ranges of the parameters $\delta$ and $\theta$ in equation (12) are $2 \leq \delta \leq 5$ and $20^{\circ} \leq \theta \leq 80^{\circ}$.

We can immediately obtain a fourth expression for the magnetic field:

$$
B \approx\left[3.68 \times 10^{-4} 10^{-0.27 \delta}(\sin \theta)^{-0.03 \delta-0.41}\left(N_{e} W\right)^{0.03 \delta-0.32} f_{p}\right]^{\frac{1}{0.03 \delta+0.6 \bar{B}}} .
$$

Considering the relation between $f_{p}$ and $t_{r}$, we can also write a fifth expression for the magnetic field from equations (1) and (12):

$$
B \approx\left[5.58 \times 10^{6} 10^{-0.27 \delta}(\sin \theta)^{-0.03 \delta-0.41}\left(N_{e} W\right)^{0.03 \delta-0.32} t_{r}^{-0.14}\right]^{\frac{1}{0.03 \delta+0.68}} .
$$


As mentioned earlier, $\sigma$ can be taken as 5 , while $L$ can be assumed to be $10^{9} \mathrm{~cm}$, thus $W$ will be $4 \times 10^{8} \mathrm{~cm}$. Moreover, the number density of the nonthermal electrons, $N_{e}$, can be generally taken to be $10^{-2}-10^{-4}$ that of the thermal electrons, i.e., one can take the value of $N_{e}=5 \times 10^{7} \mathrm{~cm}^{-3}$. Therefore, the typical value of $N_{e} W$ can be assumed to be $N_{e} W=2 \times 10^{16} \mathrm{~cm}^{-2}$.

It can be seen from equations (13) and (14) that $B$ is not sensitive to $N_{e} W$ in the valid range of $\delta$. For example, the power of $N_{e} W, 0.03 \delta-0.32$, only varies from -0.3 to -0.2 when the $\delta$ changes from 2 to 5 . So we can accurately take the factor $N_{e} W$ as $N_{e} W=2 \times 10^{16} \mathrm{~cm}^{-2}$.

Therefore, for typical values of $\delta$ (e.g. $2,3,4$, and 5) and of $\theta$ (e.g. $45^{\circ}$ and $75^{\circ}$ ), using equations (13) or (14), one can calculate the magnetic field in the source regions of microwave bursts by means of the peak frequency or the corresponding rise time obtained from observations.

\section{CONCLUDING REMARKS}

The magnetic field is both an essential and important key plasma parameter for the solar radio physics of microwave bursts. Using the analytical expressions of the magnetic field formulae derived in the present paper, and some known and relevant parameters such as the rise time, one can very conveniently calculate the magnetic field in the corona which cannot be directly measured. These magnetic field formulae, therefore, are significant not only for research on the relevant theory but also for analyses of relevant measurements.

\section{REFERENCES}

Batchelor, D.A., Crannell, C.J., Wiehl, H.J., \& Magun, A. 1985, ApJ, 295, 258 Brown, J.C., Melrose, D.B., \& Spicer, D.S. 1979, ApJ, 228, 592

Crannell, C.J., Frost, K.J., Matzler, C., Ohki, K., \& Saba, J.L. 1978, ApJ, 223, 620

Dulk, G.A. 1985, ARA\&A, 23, 169

Dulk, G.A., Melrose, D.B.,\& White, S.M. 1979, ApJ, 234, 1137

Smith, D.F. \& Lilliequist, C.G. 1979, ApJ, 232, 582

Zhao, R.-y., Magun, A., \& Schanda, E. 1990, Solar Phys., 130, 361 